\title{
Temporal integration near threshold fine structure - The role of cochlear processing
}

Epp, Bastian; Mauermann, Manfred; Verhey, Jesko L.

Link to article, DOI:

$10.1121 / 1.4799399$

Publication date:

2013

Document Version

Publisher's PDF, also known as Version of record

Link back to DTU Orbit

Citation (APA):

Epp, B., Mauermann, M., \& Verhey, J. L. (2013). Temporal integration near threshold fine structure - The role of cochlear processing. Poster session presented at 21st International Congress on Acoustics, Montreal, Canada. https://doi.org/10.1121/1.4799399

\section{General rights}

Copyright and moral rights for the publications made accessible in the public portal are retained by the authors and/or other copyright owners and it is a condition of accessing publications that users recognise and abide by the legal requirements associated with these rights.

- Users may download and print one copy of any publication from the public portal for the purpose of private study or research.

- You may not further distribute the material or use it for any profit-making activity or commercial gain

- You may freely distribute the URL identifying the publication in the public portal

If you believe that this document breaches copyright please contact us providing details, and we will remove access to the work immediately and investigate your claim 


\section{Proceedings of Meetings on Acoustics}
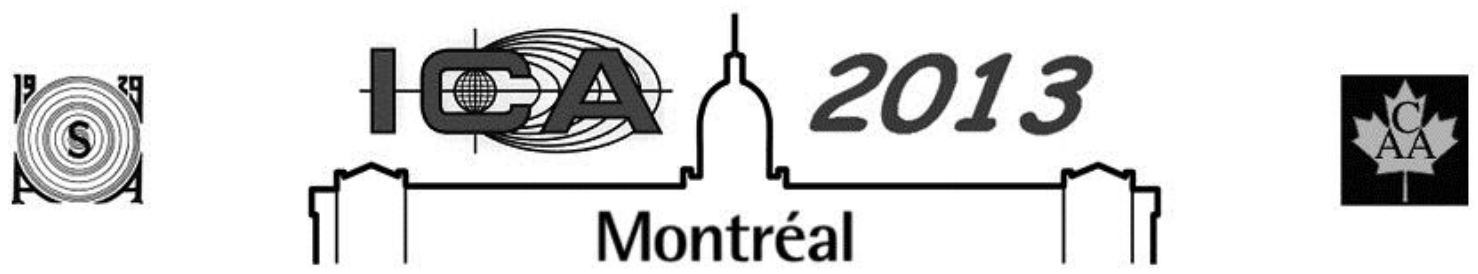

ICA 2013 Montreal

Montreal, Canada

2 - 7 June 2013

Psychological and Physiological Acoustics

Session 3aPP: Auditory Physiology and Modeling (Poster Session)

\section{3aPP27. Temporal integration near threshold fine structure - The role of cochlear processing}

Bastian Epp*, Manfred Mauermann and Jesko L. Verhey

*Corresponding author's address: Dept. of Electrical Engineering, Technical University of Denmark, Centre of Excellence for Hearing and Speech Sciences, Kgs., 2800, Lyngby, Denmark, bepp@elektro.dtu.dk

The hearing thresholds of normal hearing listeners often show quasi-periodic variations when measured with a high frequency resolution. This hearing threshold fine structure is related to other frequency specific variations in the perception of sound such as loudness and amplitude modulated tones at low intensities. The detection threshold of a pulsed tone also depends not only on the pulse duration, but also on the position of its frequency within threshold fine structure. The present study investigates if psychoacoustical data on detection of a pulsed tone can be explained with a nonlinear and active transmission line cochlea model. The model was successfully applied to other psychoacoustical data at low intensities, various types of otoacoustic emissions and physiological data. The simulations show differences in detection thresholds for tones placed in a minimum or a maximum of the fine structure, but lack a decrease of thresholds with increased pulse duration. The model was extended by including a temporal integrator which introduces a low-pass behavior of the data with different slopes of the predicted threshold curves, producing good agreement with the data. On the basis of the model simulations, it will be discussed to which extent temporal and spectral aspects contribute to the data.

Published by the Acoustical Society of America through the American Institute of Physics 


\section{INTRODUCTION}

The audiogram of normal hearing listeners, measured with a high frequency resolution, shows quasiperiodic fluctuations, referred to as threshold fine structure (e.g., Mauermann et al., 2004). In regions of fine structure, the detection threshold of pure tones can change up to around $15 \mathrm{~dB}$ over a frequency range of typically one tenth of an octave (Heise et al., 2008). Minima in the fine structure often coincide with the center frequency of spontaneous otoacoustic emissions, suggesting a common underlying mechanism (Zwicker and Schloth, 1984). It is assumed that this fine structure results from reflections of the traveling wave in the cochlea, caused by small stochastic variations of the mechanical properties of the basilar membrane (Shera, 2003). Detection thresholds of pure tones do not only depend on the frequency, but also on the tone duration. The threshold in quiet of tone bursts decreases by approximately $3 \mathrm{~dB}$ per doubling of the tone duration, i.e. when doubling the tone duration, only half of the intensity is required for the stimulus to be audible. This is in agreement with the assumption of an energy detection (intensity times duration). However, this 3 - $\mathrm{dB}$ decrease in threshold is only observed at short duration while at long durations of several hundreds of ms, thresholds hardly change with duration. This characteristic of temporal integration can be included in the energy detector approach by assuming that the intensity is lowpass-filtered and the maximum output of the lowpass filter is taken as a threshold estimate. A similar temporal integration is found in loudness data, i.e., at supra-threshold levels. At very short durations (of a few milliseconds) the temporal integration curve seems to be even steeper which can be modeled by two consecutive leaky integrators with different time constants (Poulsen, 1981). Cohen (1982) showed that fine structure also affects temporal integration, curves being steeper in fine-structure minima than in maxima. The difference in threhold is large for long durations of the tone, while thresholds are similar for small tone durations. Cohen (1982) hypothesized that differences in the temporal integration curve result from the spectral broadening associated with the decreasing duration.

In the present study, own data on detection thresholds of tone bursts are presented. In addition, a nonlinear and active transmission line model of the cochlea is used to simulate the data, in order to obtain a deeper understanding of the underlying mechanisms. The model was already successfully applied to simulate threshold in quiet, including threshold fine structure and modulation detection thresholds at low intensities (Epp et al., 2010). While the psychoacoustical data can only show the combined effect of spectral broadening and temporal integration, the simulations with the cochlea model allow to estimate the relative contribution of these two mechanism to the temporal integration data.

\section{EXPERIMENT}

\section{Methods}

Thresholds in quiet were measured for different durations of the stimulus. The stimulus frequencies were adjusted to match either a minimum ("valley condition") or a maximum ("peak condition") of the subjects individual threshold fine structure (see Fig.1). To determine individual peaks and valleys in threshold fine structure, the quiet threshold fine structure was screened in various octave bands, using a modified von Békésy tracking method as suggested by Heise et al. (2008), until a region showing sufficient fine structure was determined. A suitable pair of adjacent peak and valley was selected for each subject and used for the following experiments. All chosen frequencies lay between 1 and $3 \mathrm{kHz}$.

The tone for the main experiment (temporal integration experiment) had a duration of $8,16,32,64,128$ or $256 \mathrm{~ms}$, including a linear $4 \mathrm{~ms}$ onset ramp. At the end of the duration, the stimulus was faded out by another linear $4 \mathrm{~ms}$ ramp. Thresholds in quiet were measured using an adaptive three-interval three-alternative forced-choice paradigm with a one-up two-down rule. The silence interval between two intervals was $400 \mathrm{~ms}$. The level of the tone was reduced in steps of 6,3 and $1 \mathrm{~dB}$ after two reversal points. Thresholds were computed as the arithmetic mean over eight reversals with the smallest step size and the final estimate of the individual

threshold was the mean over three runs. Measurements were taken out in a double-walled sound attenuating booth using Sennheiser HDA200 headphones. Six normal-hearing subjects participated in the experiment. 


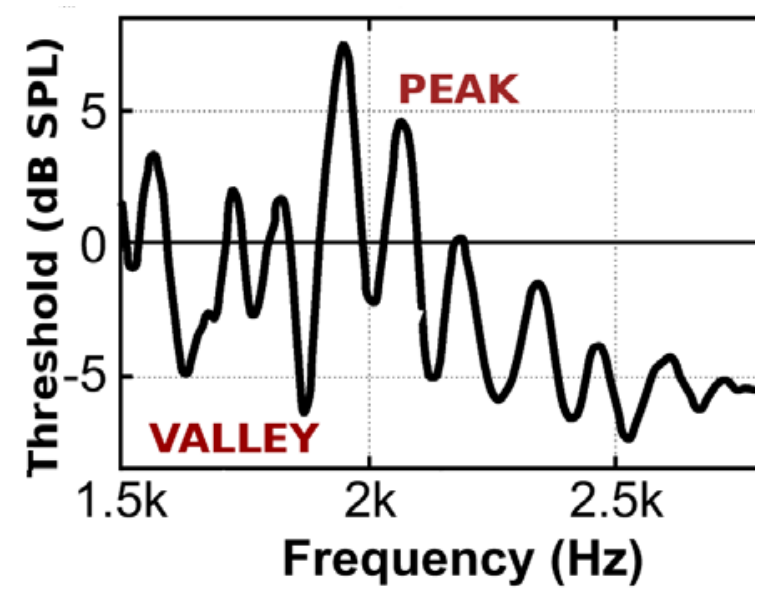

FIGURE 1: Exemplary threshold fine structure. Local maxima indicate regions of poor sensitivity (peak) and local minima regions of high sensitivity (valley). Conditions where the frequency of the test tone corresponds to a peak are referred to as "peak condition" and conditions where the frequency corresponds to a valley are be referred to as "valley condition".

\section{Results}

The mean psychoacoustical data averaged over all subjects is shown in Fig.2. Thresholds for tones in a valley or a peak decreased as the duration was increased. The difference between valley and peak was small for $8 \mathrm{~ms}$ duration and increased up to a duration of about $32 \mathrm{~ms}$. For longer durations, the difference between valley and peak was rather constant with higher threshold in the peak condition. At the longest duration of $256 \mathrm{~ms}$, this difference corresponds to the difference in the fine structure of the threshold in quiet between the corresponding threshold minima and maxima. The decrease of the data for the peak condition was about $-2 \mathrm{~dB}$ per doubling. The decrease for the valley condition was about $-4.2 \mathrm{~dB}$ per doubling for signal durations up to $64 \mathrm{~ms}$, and about $-2 \mathrm{~dB}$ per doubling for longer tone durations.

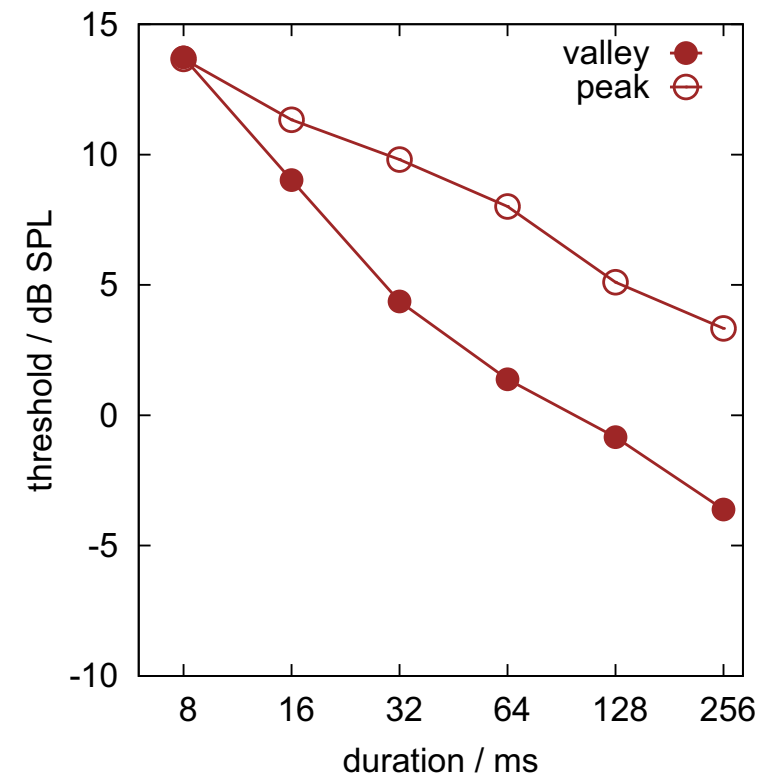

FIGURE 2: Mean thresholds over six subjects in dB SPL. The open circles indicate thresholds for the peak condition, closed circles for the valley condition. 


\section{Simulations}

\section{Model Structure}

The cochlea was modelled as a one-dimensional, non-linear and active transmission line as described in Epp et al. (2010). The model consisted of 1000 segments (cochlea partitions, CP) coupled via an incompressible fluid. Each segment $n$ can be described by the equation of motion in transverse deflection $y(n)$ :

$$
p(n)=m \ddot{y}(n)+d_{L}(n) d_{N L}(n, v) \dot{y}(n)+s(n)\left[y(n)+\left.s_{f}(n, v) y(n)\right|_{\tau}\right]
$$

with $p(n)$ denoting the pressure difference at segment $n, v$ the first derivative of $y$ with respect to time, and $m$ the mass of one CP. The damping term consisted of a linear damping term $d_{L}(n)$ and a nonlinear, velocitydependent damping term $d_{N L}(n, v)$. The stiffness was implemented by a combination of linear stiffness $s(n)$ and nonlinear, velocity-dependent feedback stiffness term $s_{f}(n, v)$.

The place-frequency map was implemented by exponential variation of the linear stiffness along the length of the cochlea. The active process in the cochlea was modeled by the combination of the nonlinear damping $d_{N L}(n, v)$ and the delayed feedback stiffness $s_{f}(n, v)$. The contribution of the active process was highest for low vibration velocities of the CP and approached zero for higher levels. This ensured linear and active processing for low input levels, compressive growth at intermediate level and linear and passive processing at high intensities. The equations of motion were solved in the time domain using a modified 4-th order Runge-Kutta method with a temporal rate of of $400 \mathrm{kHz}$. Fine structure effects were included by stochastic perturbation of the place-frequency map. With this approach, fundamental aspects of cochlear processing can be accounted for (Epp et al., 2010).

\section{Methods}

To estimate the contributions of spectral broadening and temporal integration, two simulations were performed. In a first simulation, the threshold in quiet was simulated without a temporal integration stage. Thresholds were simulated by finding the minimum level of the tone pulse required to reach an arbitrarily chosen threshold of CP velocity. This level was used as the threshold in quiet. This is the approach that was used in Epp et al. (2010) to simulate threshold in quiet. In a second simulation, a temporal integration stage was added to the output of the cochlea model. The squared velocity above a fixed, arbitrarily chosen threshold was summed across the length of the cochlea and integrated with a time constant of $200 \mathrm{~ms}$. The level of the tone pulse was increased until a critical value was reached. This critical value was adjusted to match the data for a tone duration of $8 \mathrm{~ms}$.

\section{Results}

The results of the first simulation are plotted in the left panel of Fig.3. In agreement with the data, the simulations show similar thresholds for peak and valley for a tone duration of $8 \mathrm{~ms}$. The difference between peak and valley increased up to durations of $32 \mathrm{~ms}$ and stayed constant for longer durations. The simulations show that the differences in sensitivity in a minimum or a maximum of the fine structure are small for short stimuli but become more distinct with increasing tone duration. The results of the second stimulation are plotted in the right panel of Fig.3. In line with the data, thresholds were highest for small stimulus durations and decreased with increased duration. The difference between peak and valley conditions was almost zero for $8 \mathrm{~ms}$ and increased monotonically with increased pulse duration. In contrast to the first simulations with no temporal integration stage (where the data was constant for tone durations above $64 \mathrm{~ms}$ ), the data with an included temporal integration stage decreased up the longest simulated tone duration of $256 \mathrm{~ms}$. In addition, the introduction of a temporal integrator lead to an increase in thresholds for small tone durations compared to the first simulation. 

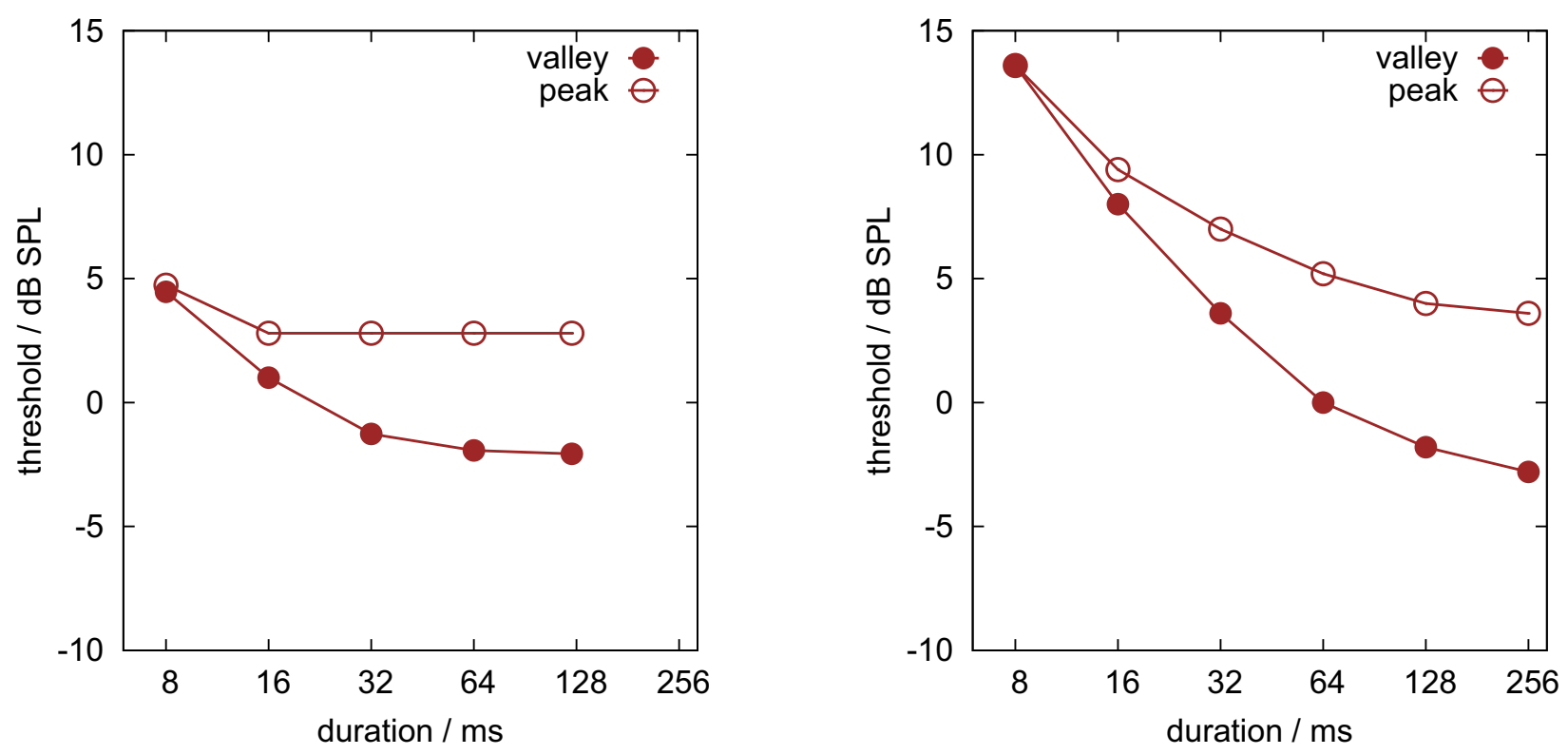

FIGURE 3: Simulation results of the cochlea model without integration stage (left panel) and with a leaky integrator as the integration stage (right panel). Open cirlces indicate data for the peak condition and closed circles for the valley condition.

\section{Discussion}

The psychoacoustical data show a close resemblance to previously published data (Cohen, 1982). Both, the data of the current study and the data by Cohen (1982) show differences in the slope of threshold decrease with tone duration for the peak and the valley condition. Cohen (1982) found a slope of about $-3.7 \mathrm{~dB}$ per doubling in the valley condition and of about $-1.7 \mathrm{~dB}$ per doubling for the peak condition. The data of the present study shows in the valley condition a decrease of about $-4.2 \mathrm{~dB}$ per doubling for tone durations up to $64 \mathrm{~ms}$ and about $-2 \mathrm{~dB}$ per doubling for longer durations. In the peak condition, the slope of $-2 \mathrm{~dB}$ per doubling is similar to the data of Cohen (1982). The slope of $-2 \mathrm{~dB}$ per doubling suggests that the linear integrator used to model the system slightly overestimates the energy available to the system for increased tone duration. In terms of energy this indicates that the integration process might not perfectly combine the energy over time. The steeper slope in the valley condition suggests that there is a contribution due to spectral broadening and temporal integration. The effect of spectral broadening decreases with increased stimulus duration, being small for tone durations where the main part of the stimulus energy is focused around the narrow frequency region of the threshold minimum. Assuming that both mechanisms play a role in temporal integration for tone bursts, the expected slope is expected to be larger then $3 \mathrm{~dB}$ per doubling due to a combined effect of temporal integration and the effect due to decreased spectral broadening.

The simulations with the cochlea model aimed to disentangle these two factors. In the first simulation without temporal integration, thresholds differed clearly between the peak and the valley conditions for long tone durations. For long durations, thresholds in the valley condition were lower than in the peak condition. For short tone durations, the differences between peak and valley conditions were smaller. This is in line with the assumption that the wider spectrum of stimuli with short duration excites broader regions along the basilar membrane. This results in less place-specific activity and a spatial integration of activity. Due to this broadened activity, adjacent regions of higher sensitivity are excited that contribute to the detection. For longer tone durations, the spectrum becomes more narrow, and hence the vibration in the cochlea more placespecific, leading to a spatially more concentrated activity. This requires a higher intensity of the stimulus to reach the threshold value in the peak compared to the valley condition.

In the second simulation, the thresholds increased due to the introduction of a temporal integrator. While in the first simulation, the vibration of the $\mathrm{CP}$ was just required to reach a threshold value, in the second simulation a critical energy was required that the tone was considered to be detected. This resulted in an increase of thresholds for all tone durations. Thresholds increased more for small than for long tone durations. The 
higher thresholds indicate that a higher stimulus level is required to reach the threshold level of integrated energy compared to longer durations where more energy can be integrated over time.

The simulations show that the combinations of spectral broadening and temporal integration leads to a slope which is larger in magnitude than for each of the two mechanisms separately, especially at short durations. In comparison to the data, the decrease is less linear. The temporal integration alone results in a purely linear slope for the data representation chosen in Fig. 2 and Fig. 3. The deviation from this linear relation is a result of the effect of spectral broadening of the stimulus within the cochlea model predictions. This predicted curvature was not found in the data. This may indicate that the shape of the simulated fine structure did not match in all details of the listeners. Especially the width of the valleys will have a large impact on the simulated data. Taking the simplicity of the mode into account, it may well be that the current approach does not include all mechanisms involved in temporal integration.

Despite these discrepancies, the current study showed that a realistic non-linear and active cochlea model combined with a simple temporal integration stage can account for the main aspects of the effect of fine structure on temporal integration.

\section{ACKNOWLEDGMENTS}

We thank the Deutsche Forschungsgemeinschaft (Grant Nos. SFB/TRR31 and KO942/18-1\&2) for their support of the project and Heiner Lendzian for collecting the data in our lab.

\section{REFERENCES}

Cohen, M. (1982). "Detection threshold microstructure and its effect on temporal integration data.", J. Acoust. Soc. Am. 71, 405-409.

Epp, B., Verhey, J. L., and Mauermann, M. (2010). "Modeling cochlear dynamics: interrelation between cochlea mechanics and psychoacoustics.”, J. Acoust. Soc. Am. 128, 1870-83.

Heise, S. J., Verhey, J. L., and Mauermann, M. (2008). “Automatic screening and detection of threshold fine structure.", Int. J. Audiol. 47, 520-32.

Mauermann, M., Long, G. R., and Kollmeier, B. (2004). "Fine structure of hearing threshold and loudness perception”, J. Acoust. Soc. Am. 116, 1066.

Poulsen, T. (1981). “Loudness of tone pulses in a free field.”, J. Acoust. Soc. Am. 69, 1786-90.

Shera, C. (2003). "Mammalian spontaneous otoacoustic emissions are amplitude-stabilized cochlear standing waves", J. Acoust. Soc. Am. 114, 244.

Zwicker, E. and Schloth, E. (1984). "Interrelation of different oto-acoustic emissions”, J. Acoust. Soc. Am. 75, 1148-1154. 